\title{
Hybrid Anion Exchange Hollow Fiber Membrane for Delivery of Ionic Drugs
}

\author{
Na Wang, ${ }^{1}$ Mengbing Cui, ${ }^{1}$ Cuiming Wu, ${ }^{2}$ Yiyun Cheng, ${ }^{3}$ and Tongwen $\mathrm{Xu}^{1}$ \\ ${ }^{1}$ Laboratory of Functional Membranes, School of Chemistry and Material Science, University of Science and Technology of China, \\ Hefei 230026, China \\ ${ }^{2}$ School of Chemical Engineering, Hefei University of Technology, Hefei 230009, China \\ ${ }^{3}$ School of Life Sciences, East China Normal University, Shanghai 200062, China \\ Correspondence should be addressed to Tongwen Xu, twxu@ustc.edu.cn
}

Received 30 October 2011; Revised 29 December 2011; Accepted 10 January 2012

Academic Editor: Victor V. Nikonenko

Copyright ( $) 2012$ Na Wang et al. This is an open access article distributed under the Creative Commons Attribution License, which permits unrestricted use, distribution, and reproduction in any medium, provided the original work is properly cited.

Hybrid anion exchange hollow fiber membranes (HAEHFMs) based on bromomethylated poly(2,6-dimethyl-1,4-phenylene oxide) (BPPO) are proposed as potential drug carriers for four anionic model drugs, including the sodium salts of benzoate (NaBS), salicylate (NaSA), meta-amino salicylate (NaMAS), and loxoprofen (NaLS). The results of the static loading and release experiments suggest that electrostatic interaction, hydrogen bonding, and hydrophobic interaction are the main interaction patterns between the membrane and the drugs. And they are directly influenced by the external phase conditions and the drug physicochemical characteristics, such as structure, molecular weight, dissociation (pKa), and hydrogen bonding capability. Among the four different drugs, NaSA and NaMAS appear to be the most suitable for controlled release by the HAEHFM due to their excellent adsorption/release behaviors.

\section{Introduction}

Controlled drug release has attracted increasing attention during recent decades because of its special advantages on providing a relatively steady blood level of drug, optimizing the drug therapeutics and improving patient compliance. The most significant pursuit of developing controlled release system is to prolong the retention of the drug and get a constancy of release rate. Membranes have been evaluated as one of the most promising drug delivery vehicles $[1,2]$. In order to develop rate-limiting drug delivery membrane, ion transport and ion exchange mechanisms can be introduced through the use of ion exchange membranes (IEMs). The ion exchange groups of the IEMs can capture the ionized drugs through electrostatic interactions and thus provide a permselectivity for the therapeutic agents in the drug delivery profiles [3]. Up to date, many types of IEMs have been used directly or modified as drug vehicles such as Nafion [4], modified chitosan [1, 5-7], polypyrrole [8], poly(acrylic acid) grafted poly(vinylidene fluoride) (PAA-PVDF) [9, 10], and bromomethylated poly(2,6-dimethyl-1,4-phenylene oxide) (BPPO) membranes [11]. The rate-limiting performance of the IEMs for ionic drugs mainly depends on the physical and chemical properties of the IEMs and the drugs, as well as the external solution conditions [12].

IEMs can be classified into ion exchange flat membranes and ion exchange hollow fiber membranes (IEHFMs) according to the physical configuration. IEHFMs, as a novel type of drug delivery vehicles, have been proved to be effective in rate-limiting drug release. Our research group has previously promoted a novel type of hybrid anion exchange hollow fiber membrane (HAEHFM) from brominated poly (2,6-dimethyl-1,4-phenylene oxide) (BPPO) and $\gamma$-methacryloxypropyl trimethoxysilane ( $\gamma$-MPS) for controlled release of sodium salicylate (NaSA) [13]. The HAEHFM exhibits low toxicity, large effective surface area, and high ion exchange content (IEC), ensuring a high drug loading efficiency $(28.4 \%)$ and a relatively low release rate (drug released in 7 days under physiological condition: 51.9\%).

The present work is to extend our previous study and to investigate further the effect of the drug characteristics on the loading and release behaviors. As well known, 
TABLE 1: Structures and physicochemical characteristics of the model drugs.

\begin{tabular}{|c|c|c|c|c|}
\hline Compound & Structure & Mw & $\mathrm{pKa}^{17-20}$ & HBD/HBA* \\
\hline Sodium benzoate (NaBS) & & 144.1 & $4.17(-\mathrm{COOH})$ & $0 / 2$ \\
\hline Sodium salicylate (NaSA) & & 160.1 & $\begin{array}{c}2.98(-\mathrm{COOH}) \\
13.9(-\mathrm{OH})\end{array}$ & $1 / 3$ \\
\hline Sodium meta-amino salicylate (NaMAS) & & 175.1 & $\begin{array}{c}2.74(-\mathrm{COOH}) \\
5.84\left(-\mathrm{NH}_{3}^{+}\right) \\
13.9(-\mathrm{OH})\end{array}$ & $1 / 4$ \\
\hline Loxoprofen sodium (NaLS) & & 268.3 & $4.20(-\mathrm{COOH})$ & $0 / 3$ \\
\hline
\end{tabular}

\footnotetext{
${ }^{*}$ Hydrogen bond donor (based on -OH groups)/hydrogen bond acceptor (based on O-atoms and N-atoms); it is assumed that carboxyl groups are ionized
} during the experiments.

the physicochemical properties of the drugs such as charge, molecular weight, dissociation ( $\mathrm{pKa}$ ), lipophilicity and hydrogen bonding can greatly affect the adsorption and release behaviors [12]. Previous researches are abundant regarding the effect of these drug properties on the controlled release behavior of ion exchange flat membranes, fibers, or resins [12, 14-16]. For example, Jaskari et al. and Hanninen et al., both, proved that lipophilic drugs tend to bond onto the weak base anion exchange fibers $[15,16]$. However, few studies have been performed with IEHFMs. For our IEHFMs, the interaction mechanisms with the drug might be even more complex, since there are both organic and inorganic components in the membrane matrix. The presence of $-\mathrm{SiOH}$ and ester groups from $\gamma$-MPS may influence the loading and release behaviors comprehensively.

Four model drugs are selected here, including the sodium salts of benzoate (NaBS), salicylate (NaSA), meta-amino salicylate (NaMAS), and loxoprofen (NaLS) (Table 1 [1720]). Among these, salicylic acid (SA), meta-amino salicylate (MAS), and loxoprofen (LS) are widely used nonsteroidal anti-inflammatory drugs (NSAIDs). NaBS, a commonly used food preservative, is also used here for its structure similarity to the other drugs. The four drugs differ in physicochemical characteristics such as molecular weight, $\mathrm{pKa}$, and hydrogen bonding capacity. Nevertheless, they all contain carboxyl groups, which produce negatively charged anions when dissociated. The drug loading capacity and the drug release rate and extent from the HAEHFM in different external solutions are to be studied and compared. The mechanisms of drug binding into and release from the ion exchange drug carriers are to be discussed so as to provide guidance for the selection, optimization, and application of the drug vehicles.

\section{Material and Methods}

2.1. Materials. Brominated poly(2,6-dimethyl-1,4-phenylene oxide) (BPPO) hollow fiber membranes were kindly supplied by Tianwei Membrane Corporation Ltd. (Shandong, China) with 53\% benzyl substitution ratio and 47\% aryl substitution ratio. $\gamma$-methacryloxypropyl trimethoxysilane $(\gamma$ MPS) and triethylamine (TEA) were diluted with ethanol to $1.0 \mathrm{~mol} / \mathrm{L}$ before use. Sodium salicylate (NaSA) and sodium meta-amino salicylate (NaMAS) were purchased from Tianjin Damao Chemical Reagent Factory (Tianjin, China) and Weihai Disu Pharmaceutical Co., Ltd. (Shandong, China), respectively. Sodium benzoate (NaBS), loxoprofen sodium (NaLS), and dialysis membrane (molecular weight cut-off, MWCO $3500 \mathrm{Da}$ ) were obtained from Bio Basic Inc. (BBI) (Shanghai, China). Bovine serum albumin (BSA) and glucose (Glc) were from Sinopharm Chemical Reagent Co., Ltd (Shanghai, China). The reagents were all of analytical grade and double-distilled water was used throughout. 


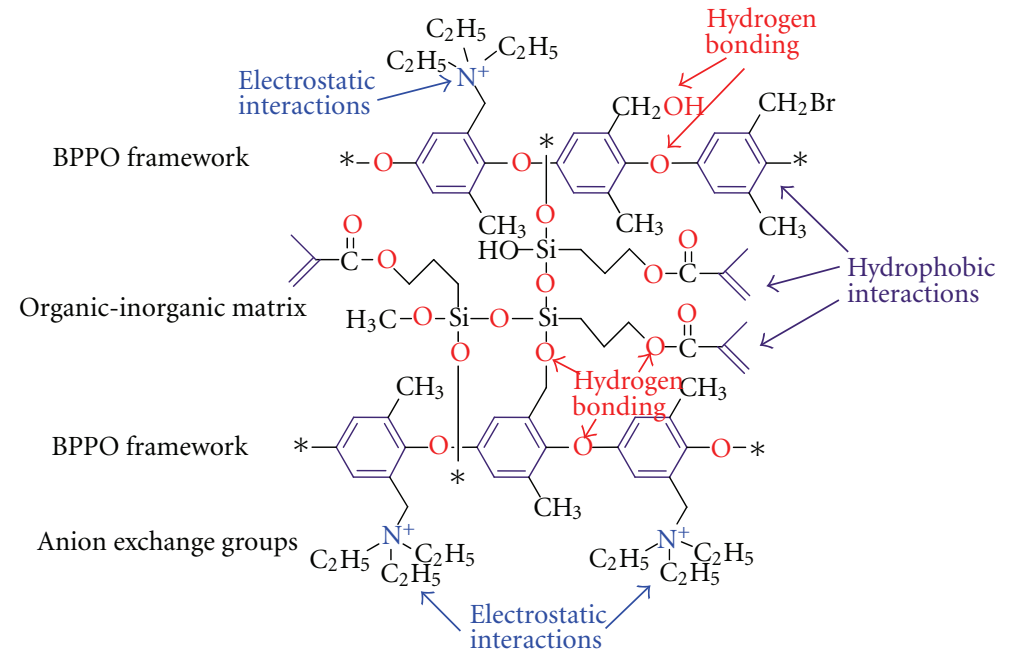

FIgURE 1: The structure of the HAEHFM and different interaction patterns between the HAEHFM and drugs.

Phosphate buffer solutions (PBSs) with $\mathrm{pH}$ values of 5.0, 7.4, and 9.0 were prepared by $\mathrm{Na}_{2} \mathrm{HPO}_{4}-\mathrm{KH}_{2} \mathrm{PO}_{4}$ with phosphate concentration of $0.2 \mathrm{~mol} / \mathrm{L}$. One simulated body fluid was prepared containing $0.2 \mathrm{~mol} / \mathrm{L}$ PBS $(\mathrm{pH}=7.4)$, $100 \mathrm{mg} / \mathrm{dL}$ glucose, and $70 \mathrm{mg} / \mathrm{mL}$ BSA. The simulated body fluid without BSA was also prepared.

\subsection{Preparation and Characterizations of the Drug Carrier:} The HAEHFM. The HAEHFM was prepared from BPPO hollow fiber membrane through three steps, as has been described in detail in our earlier study [21]: partial hydrolysis by $\mathrm{KOH}$, in situ sol-gel process with $\gamma$-MPS, and quaternization by TEA. The main properties of the HAEHFM are as follows: inner and outer diameter of $0.70 \mathrm{~mm}$ and $1.06 \mathrm{~mm}$, ion exchange capacity (IEC) of $1.76 \mathrm{mmol} / \mathrm{g}$ dry membrane, water content $\left(W_{R}\right)$ of $62.9 \%$, and dimensional change ratio (DCR) of $12.9 \%$ in water.

As illustrated in Figure 1, the BPPO frameworks are crosslinked by Si-O-Si network formed in the sol-gel process. According to the polymer structure, three main types of interaction patterns between the HAEHFM and drugs can be present: electrostatic interactions, hydrogen bonding, and hydrophilic interactions. The strong anion exchange groups (quaternary amino groups) on the backbone of the polymer provide sites for electrostatic binding. The oxygen atoms (from BPPO frameworks, ester bonding in $\gamma$-MPS, and the Si-O-Si bonding) and the hydroxyls groups (from BPPO and silanol groups) can form hydrogen bonds with drugs in water. Besides, hydrophilic interactions may exist between drugs and the benzene rings or methyl vinyls of the HAEHFM.

2.3. Drug Loading Studies. The HAEHFM was loaded with the model drugs through static adsorption. Dry membranes $(0.05 \mathrm{~g})$ were cut into pieces with length of $5-8 \mathrm{~mm}$ then added into $4 \mathrm{~mL}$ aqueous solutions of different drugs (from
TABLE 2: The UV test parameters of different drugs.

\begin{tabular}{lcc}
\hline Drug & Wavelength $(\mathrm{nm})$ & Test concentration $(\mu \mathrm{mol} / \mathrm{L})$ \\
\hline NaBS & 228 & $4-114$ \\
NaSA & 295 & $27-266$ \\
NaMAS & 330 & $27-11500$ \\
NaLS & 225 & $12-132$ \\
\hline
\end{tabular}

0.05 to $100 \mathrm{mmol} / \mathrm{L}$ ) and kept at $30^{\circ} \mathrm{C}$ for $24 \mathrm{~h}$. Then the membrane was taken out and washed by water (with a total volume of $5 \mathrm{~mL}$ ) to remove the drug adhered on the surface. The washing solutions and the adsorption solutions after drug loading were collected for concentration analysis.

For concentration determination, the drug solutions were diluted into proper concentrations and then tested using a UV-2550 UV-VIS spectrophotometer (Kyoto, Japan). The characteristic adsorption wavelength of each drug was found and listed in Table 2. Because there is a linear relationship between the absorbance and concentration when the absorbance is between $0.1-1.0$, each sample was diluted to proper concentration before testing. The testing concentration ranges of the model drugs are also listed in Table 2. The drug loading content was calculated by the following equation:

$$
S=\frac{V\left(c_{0}-c\right)}{m} \times 10^{-3}
$$

where $S(\mathrm{mmol} / \mathrm{g}$ dry membrane) is the total content of a model drug adsorbed by the HAEHFM; $c_{0}$ and $c(\mathrm{mmol} / \mathrm{L})$ are the concentrations of the model drug before and after drug loading. $V(\mathrm{~mL})$ means the volume of the adsorption solution, which is $4 \mathrm{~mL}$ in this study. $m(\mathrm{~g})$ means the weight of the testing membrane, which is $0.05 \mathrm{~g}$ in this study. 
The curves of the loading contents versus original drug concentrations are fitted by the Langmuir isotherm mechanism [22]:

$$
q=\frac{q_{m} K c_{0}}{1+K c_{0}}
$$

where $K$ is Langmuir equilibrium constant ( $\mathrm{mL} / \mathrm{mg}), q$ is the drug adsorption under a certain original drug concentration, and $q_{m}$ is the maximum amount of drug adsorption. By cur ve fitting of the drug loading data under different drug con centrations, the value of $q_{m}$ and $K$ can be calculated.

2.4. Release Behaviors. Three different release experiments were performed for each model drug.

(1) The release experiment in pure water. The HAEHFMs $(0.05 \mathrm{~g})$ loaded with drugs were immersed into $50 \mathrm{~mL}$ of water and kept at $37^{\circ} \mathrm{C}$ for $12 \mathrm{~h} .3 \mathrm{~mL}$ of the solution was sampled at each predetermined sam pling time. Fresh water was added immediately after each sampling. The cumulative amount of drug re leased was determined by the UV absorbance.

(2) The $\mathrm{pH}$ dependence. The procedures were the same as above in pure water, except that $0.2 \mathrm{~mol} / \mathrm{L}$ PBSs with varied $\mathrm{pH}$ values $(5.0,7.4$, and 9.0) were used.

(3) Release behaviors under physiological condition. The HAEHFM loaded with the model drug was added into a dialysis bag containing $5 \mathrm{~mL}$ simulated body fluid medium. Then the dialysis bag was immediately immersed into the simulated body fluid without BSA $(45 \mathrm{~mL}) .3 \mathrm{~mL}$ of the solutions out of the dialysis bag were sampled every $12 \mathrm{~h}$ and the experiments last for one week.

Diffusion coefficients $D\left(\mathrm{~cm}^{2} / \mathrm{s}\right)$ can be obtained through fitting the release curves by the Higuchi equation [23]:

$$
\frac{Q}{A}=2 C_{0} \sqrt{\frac{D t}{\pi}},
$$

where $Q / A$ is the amount of drug released per unit area the time $t$ and $C_{0}$ is the initial concentration of a model drug in the HAEHFM. $A$ is the membrane surface area and can be calculated from the inner, outer diameter, and the length of the membrane.

\section{Results and Discussion}

3.1. Binding of Different Drugs to the AEHFM. The data of the drug loading contents versus different original drug concentrations are fitted by Langmuir isotherm mechanism (2). Figure 2 suggests that the data of NaSA, NaMAS, and NaLS can fit the Langmuir isotherm mechanism well (the coefficient of determination $\left.\left(R^{2}\right)>0.97\right)$. This indicates that the adsorption is congruous to the assumptions of the Langmuir isotherm mechanism: (1) the drug does not interact with itself; and (2) only one monolayer is formed at the maximum adsorption [22]. This way of adsorption is beneficial for slow and sustained drug release.

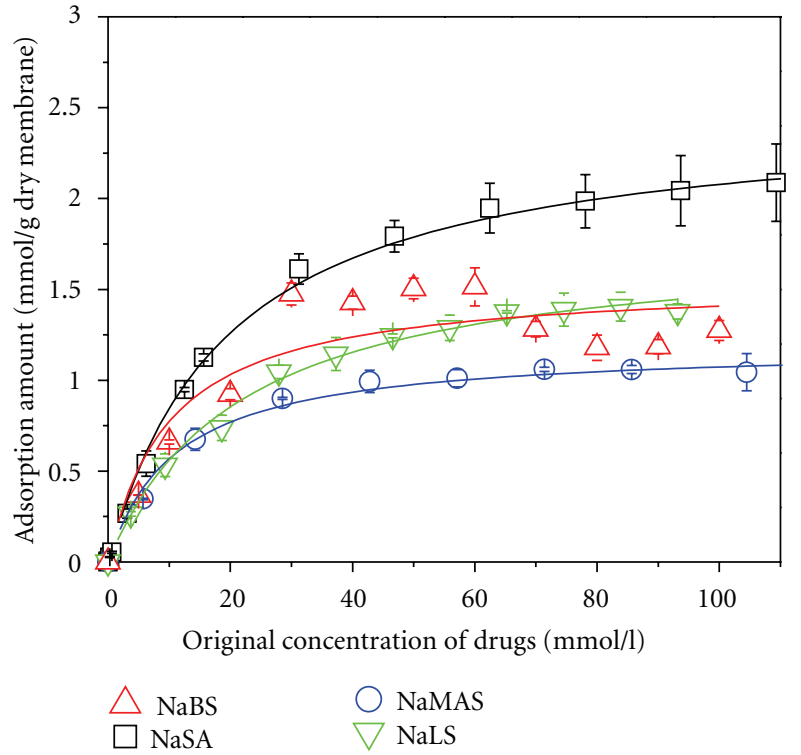

FIGURE 2: Loading contents of different drugs onto the HAEHFM as the original drug concentrations increase. Experimental conditions: $24 \mathrm{~h}$ at $30^{\circ} \mathrm{C}$.

Nevertheless, the adsorption behaviour of NaBS does not agree well with the fitting result $\left(R^{2}=0.73\right)$. The adsorption amount of NaBS increases first with the increase of $\mathrm{NaBS}$ concentration, then decreases somehow when $\mathrm{NaBS}$ concentration is in the range of $60-80 \mathrm{mmol} / \mathrm{L}$, and finally increases again at $\mathrm{NaBS}$ concentration higher than $80 \mathrm{mmol} /$ L. The abnormity of the NaBS adsorption behavior should be due to the peculiarity of the drug structure. As shown in Table 1, NaBS molecule possesses only one carboxyl group which is highly hydrophilic. The benzene ring, on the other hand, is highly hydrophobic. Hence phenyl stackings might be formed in aqueous solution through the parallel displaced or T-shaped arrangements [24] as shown in Figure 3. The possible explanation of the changing trend of the NaBS curve in Figure 2 can be presumed as follows. Under low concentration of $\mathrm{NaBS}$, the drug adsorption amount onto the membrane increases with the NaBS concentration as the number of free drug molecules in the solution increases. However, when $\mathrm{NaBS}$ concentration is higher than $60 \mathrm{mmol} / \mathrm{L}$, NaBS stackings begin to form, which decrease the number of free drug molecules and thereby the adsorption amount decreases. With further increase in NaBS concentration, the stackings grow in size and number and further formation of the stacking structure becomes more difficult. Therefore, the number of free drug molecules becomes increasing again, leading to an increase in drug adsorption amount.

The $q_{m}$ values of the drugs are obtained from fitting the curves of the loading contents versus original drug concentrations by equation (2) and the results are illustrated in Figure 4 . The $q_{m}$ values are all over $1.2 \mathrm{mmol} / \mathrm{g}$, relatively high as compared with some reported values [11, 25]. Meanwhile, the value of $q_{m} /$ IEC, which indicates the ratio of maximal drug binding content and the ion exchange capacity of the membrane, is in the range of $68-141 \%$. The $q_{m} /$ IEC 


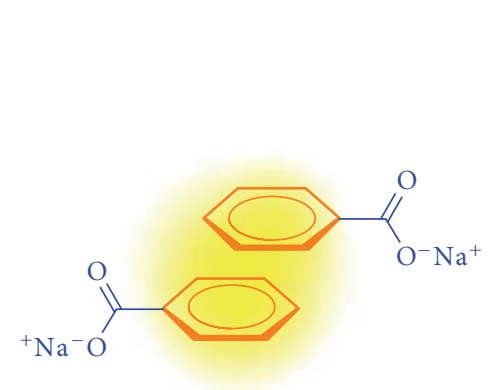

(a) Parallel displaced

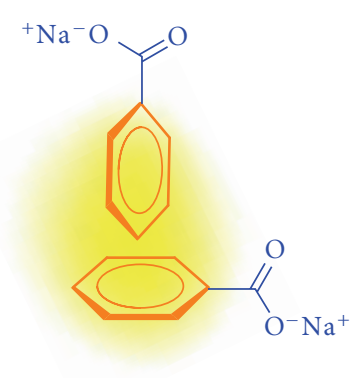

(b) T-shaped

FIgURE 3: The supposed arrangements of the stackings formed in NaBS solution.

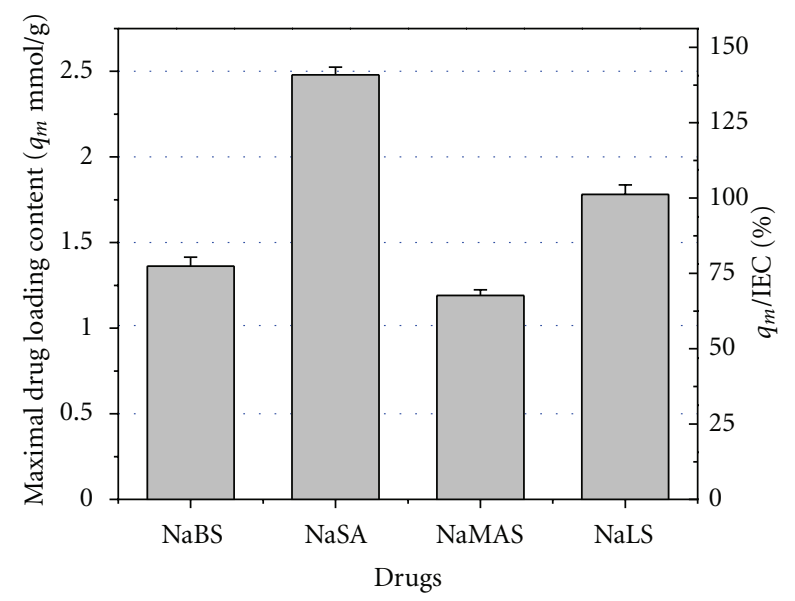

FIGURE 4: The maximal drug loading contents $\left(q_{m}\right)$ and the values of $q_{m}$ /IEC for different drugs onto the HAEHFM.

values of NaSA and NaLS are over 100\%, indicating that some other interactions besides the electrostatic interaction are present.

The $q_{m}$ values of the drugs can be arranged as NaSA $>$ NaLS $>$ NaBS $>$ NaMAS. The reason should be attributed to the difference of the drug structures, as explained in the following.

(1) Compared with NaBS and NaLS, salicylate (NaSA and NaMAS) possesses an extra phenolic hydroxyl group ( $\mathrm{Ph}-\mathrm{OH})$. The $\mathrm{Ph}-\mathrm{OH}$ group can partly dissociate into phenol anion $\left(\mathrm{Ph}-\mathrm{O}^{-}\right)$and form hydrogen bonding with the drugs, both of which are beneficial for the affinity of the drug to the membrane. And the pKa values of NaSA and NaMAS are 2.98 and $2.74(-\mathrm{COOH})$, much lower than the other two drugs (Table 1). Therefore, the high $q_{m}$ value of NaSA can be well understood.

(2) Nevertheless, NaMAS contains an amino group, which might be protonated into $-\mathrm{NH}_{3}{ }^{+}$group. Hence, neutral intrinsic molecular salts can be formed in NaMAS solution $[12,26]$, decreasing the adsorption of NaMAS to the membrane. Therefore, the $q_{m}$ value of NaMAS is the lowest.
(3) NaLS possesses one more carbonyl and one fivemembered ring as compared with $\mathrm{NaBS}$. The $\mathrm{O}$-atom from the carbonyl can act as the hydrogen bonding acceptor, and the five-membered ring increases the hydrophobility of NaLS. Hence the hydrogen bonding and hydrophobic interactions between NaLS and the membrane are increased, leading to higher $q_{m}$ value of NaLS than NaBS.

\subsection{Release of Different Drugs from the AEHFM}

3.2.1. Release Behaviors of Different Drugs in Pure Water. The release behavior of the model drugs from the HAEHFM in pure water was examined and the result is shown in Figure 5. Only $0.85-7.24 \%$ of the entrapped drugs are released during the period of $12 \mathrm{~h}$. The diffusion coefficients $(D)$ of the drugs are calculated to be $4.7 \times 10^{-7}-3.5 \times 10^{-5} \mathrm{~cm}^{2} / \mathrm{s}$ according to Higuchi equation (3), as listed in Table 3. The $D$ values of NaSA and NaMAS are relatively low as compared with some reported values $[12,27]$, indicating the high potential of NaSA and NaMAS for controlled release by HAEHFM. As for NaBS, the $D$ value is higher than some reported values. For instance, the $D$ value of NaBS from liquid crystalline gels was $0.12 \times 10^{-7} \mathrm{~cm}^{2} / \mathrm{s}$, and the release percentage within $24 \mathrm{~h}$ was $25.2 \%$ [28]. The significant difference is mainly due to the difference of materials properties and the drug loading content. The release behaviour of NaLS has been rarely reported in the literature and hence the data here can give clues for its future research.

The release rates of the different model drugs (see Figure 5 or Table 3) decrease according to the following sequence: NaLS > NaBS > NaSA > NaMAS. Possible reasons for the change trend can be explained as follows.

(1) The $D$ values of salycilates (NaSA and NaMAS) are much lower than the other two drugs. This should be mainly due to their higher affinity to the membrane, since phenolic hydroxyl group ( $\mathrm{Ph}-\mathrm{OH})$ can provide additional electrostatic interaction and hydrogen bonding.

(2) The $D$ value of NaMAS is the lowest among all the drugs. As discussed in Section 3.1, intrinsic molecular salts can be formed in NaMAS solution because 
TABLE 3: Diffusion coefficients $\left(D / \times 10^{-6} \mathrm{~cm}^{2} / \mathrm{s}\right)$ of different drugs from $\mathrm{BPPO}-\gamma$-MPS $(+)$ hollow fiber membrane at $37^{\circ} \mathrm{C}$ under different outer phase conditions, calculated by the Higuchi equation (3).

\begin{tabular}{lcccc}
\hline $\begin{array}{l}\text { Outer phase } \\
\text { conditions }\end{array}$ & NaBS & NaSA & NaMAS & NaLS \\
\hline Pure water & 16.79 & 1.456 & 0.4714 & 35.44 \\
PBS at pH 5.0 & 1483 & 218.8 & 100.8 & 1598 \\
PBS at pH 7.4 & 1327 & 123.9 & 77.42 & 4531 \\
PBS at pH 9.0 & 1429 & 39.36 & 345.0 & 3803 \\
PBS with $100 \mathrm{mg} / \mathrm{dL}$ & & & & \\
Glc and 70 mg/mL & 161.7 & 132.1 & 72.61 & 353.6 \\
BSA, pH 7.4 & & & & \\
\hline
\end{tabular}

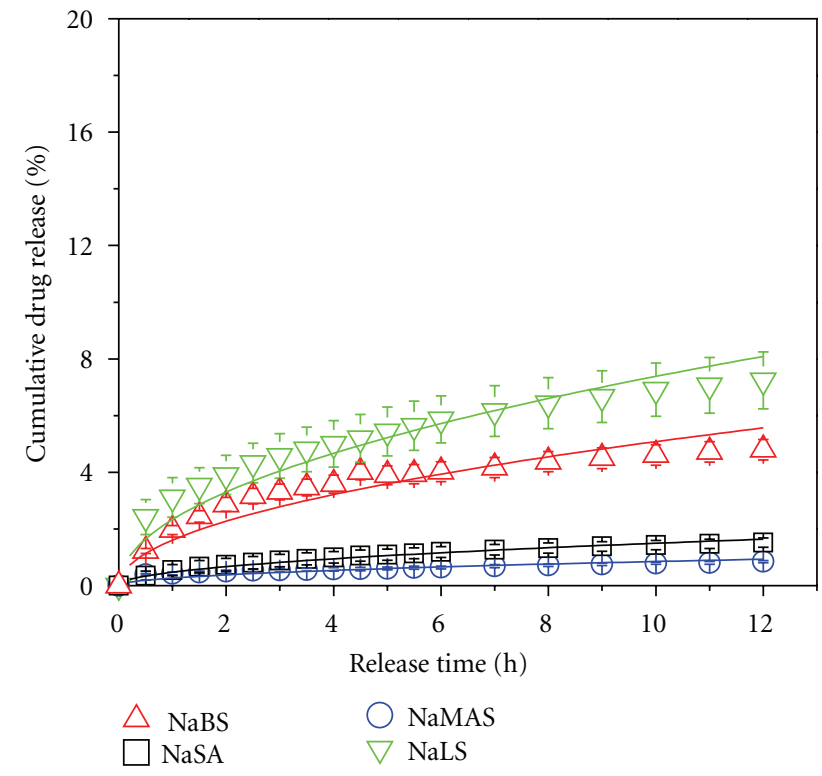

FIgURE 5: Release behaviors of different drugs from the HAEHFM in pure water at $37^{\circ} \mathrm{C}$.

of the presence of $-\mathrm{NH}_{2}$ groups. The electrostatic interaction with the membrane is diminished. On the other hand, the hydrogen bonding capacity of NaMAS is the strongest among all the drugs as illustrated in Table 1. Therefore, the low release rate of NaMAS should be mainly attributed to the hydrogen bonding by both $-\mathrm{OH}$ group and $-\mathrm{NH}_{2}$ groups.

(3) NaLS shows the largest release rate in pure water, indicating the least stable association between NaLS and the membrane. As discussed in Section 3.1, NaLS contains five-membered ring and hence the hydrophobic interaction plays more important role for its affinity to the membrane. The hydrophobic interaction is relatively weak as compared with electrostatic and hydrogen bonding interactions. Besides, the fivemembered ring increases the steric hindrance of NaLS. Therefore, the release rate of NaLS is the highest among all the drugs.
3.2.2. Release Behaviors of Different Drugs at Varied $p H$ Values. Figure 6 illustrates the release behaviors of the model drugs in acidic ( $\mathrm{pH}$ 5.0, Figure 6(a)), neutral ( $\mathrm{pH} 7.4$, Figure 6(b)), and basic (pH 9.0, Figure 6(c)) conditions. The $D$ values are calculated and listed in Table 3 . The $D$ values are in the range of $3.9 \times 10^{-5}-4.5 \times 10^{-3} \mathrm{~cm}^{2} / \mathrm{s}$, much higher than the values in pure water. Hence, the increase of ion concentration in the external phase can lead to a significant increase in the drug release rate $[13,29]$. As promoted by Hanninen, the Donnan potential is decreased, and the molar amounts of counterions, as well as the concentration gradient, are increased when salty solutions are used [29]. Besides, the hydrogen bonding can also be disturbed by salty environment. All the changes can result into a higher release rate of the drugs.

At $\mathrm{pH} 5.0$ and $\mathrm{pH} 7.4$, the $D$ values follow the same changing trend as in pure water: NaLS > NaBS > NaSA > NaMAS. Nevertheless, the $D$ value of NaMAS is higher than that of NaSA at $\mathrm{pH}$ 9.0. This can be explained as follows. NaMAS contains additional $-\mathrm{NH}_{2}$ groups and hence the hydrogen bonding plays important role in the affinity of NaMAS to the membrane. Hydrogen bonding tends to form at lower $\mathrm{pH}$ value, especially around the $\mathrm{pKa}$ value of the drug [30]. In basic condition, the hydrogen bonding can be destroyed and hence the release rate is increased.

As for the influence of the $\mathrm{pH}$ values on the $D$ values of each drug, interesting phenomena can be found from Table 3 as follows.

(1) The $D$ value of NaBS is not significantly affected by the $\mathrm{pH}$ value. $\mathrm{NaBS}$ has $\mathrm{pKa}$ value of 4.17 and hence is negatively charged in different $\mathrm{pH}$ conditions (5.0, 7.4, and 9.0). The main interaction between NaBS and the membrane is electrostatic interaction. Thus the release behavior of $\mathrm{NaBS}$ is relatively stable when $\mathrm{pH}$ changes.

(2) The $D$ value of NaSA decreases gradually with the increase of the $\mathrm{pH}$ values. The reason for this changing trend has been discussed in our previous work [13]. Namely, the phenolic hydroxyls (Ph-OH) of NaSA can be partially deprotonated at higher $\mathrm{pH}$ condition. Therefore, the electrostatic interaction with the membrane is enhanced.

(3) The D value of NaMAS at pH 9.0 is abnormally larger than that at the other $\mathrm{pH}$ conditions. This is due to the destruction of the hydrogen bonding, as discussed previously.

(4) The $D$ value of NaLS at pH 5.0 is relatively lower than the values at the other $\mathrm{pH}$ conditions. This should be due to the strengthening of the hydrogen bonding since pH 5.0 is near the pKa value of NaLS (4.20) [30].

3.2.3. Release Behaviors of Different Drugs in a Simulated Body Fluid. As the HAEHFM is researched for the potential use of drug delivery to the focus in human body, the release behaviors of the drugs under physiological condition have been investigated. The curves in Figure 7 are fitted according to Higuchi equation (3) and the diffusion coefficients $(D)$ 


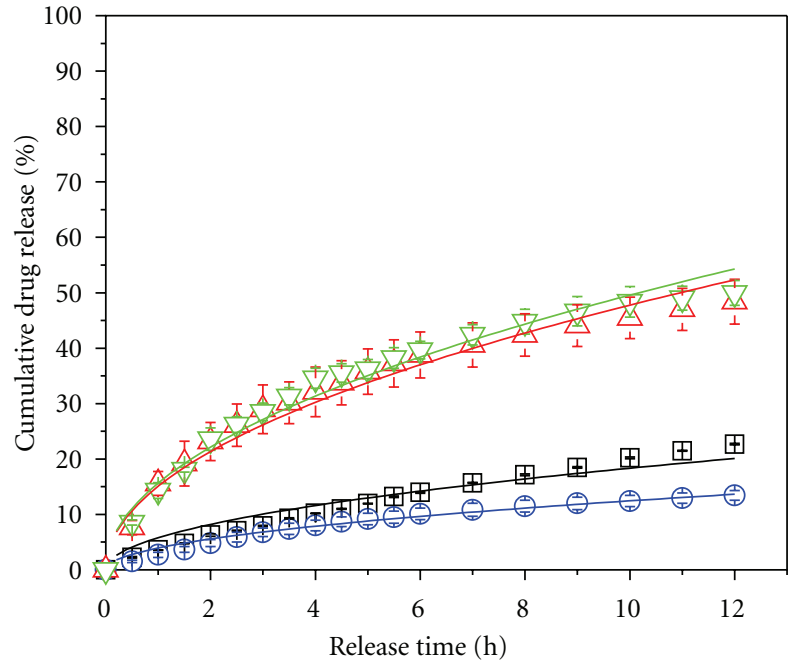

(a)

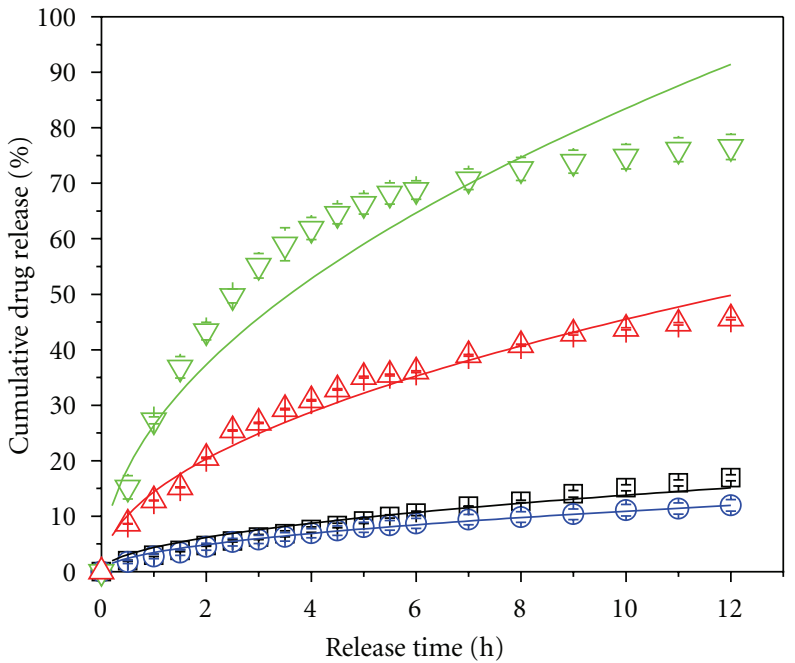

(b)

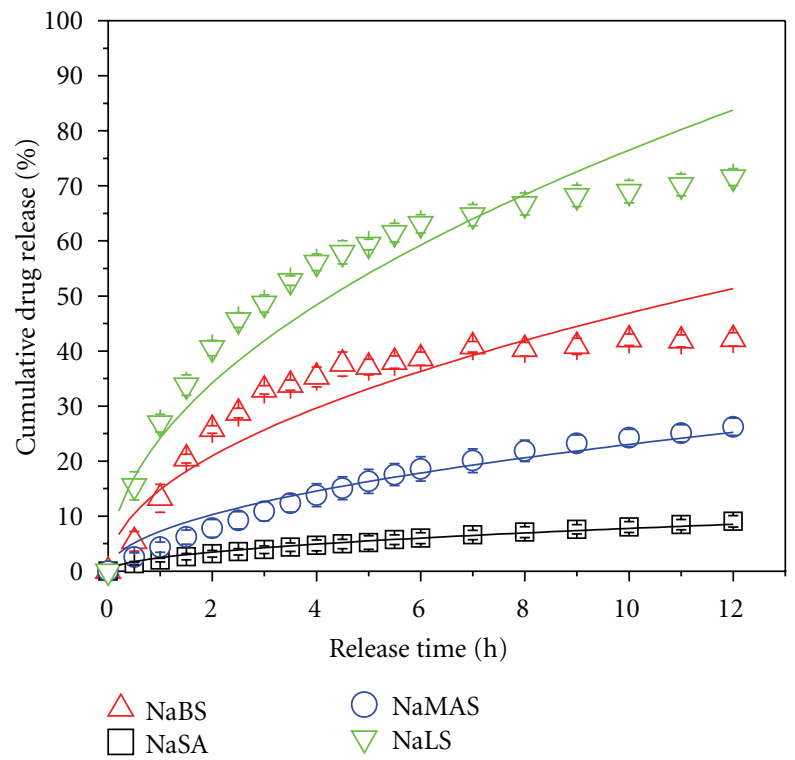

(c)

Figure 6: Release behaviors of different drugs from the HAEHFM at $37^{\circ} \mathrm{C}$ at (a) $\mathrm{pH} 5.0$, (b) $\mathrm{pH} 7.4$, and (c) $\mathrm{pH} 9.0$.

are listed in Table 3. The release curves of NaSA and NaMAS can fit the equation well and the corresponding $D$ values are similar to the values in $\mathrm{PBS}$ at $\mathrm{pH}$ 7.4. However, the curves of $\mathrm{NaBS}$ and NaLS in the simulated body fluid deviate from the Higuchi equation $\left(R^{2}=0.35\right.$ and 0.74$)$. This indicates that the release mechanisms of these two drugs do not accord with the assumptions of Higuchi equation. Three possible phenomena can be presented as the reasons: (i) there are protein and sugar in the solution, and hence the solubility of the drugs is decreased and the solution is not under a perfect dissolution condition; (ii) a constant diffusivity cannot be maintained as the viscosity of the solution is relatively high; (iii) the swelling of the polymer cannot be neglected. The size of NaLS molecule is the largest among all the drugs, and NaBS may form stackings due to the hydrophilic-hydrophobic interactions. Hence the three possible phenomena, especially the former two, have more significant influence on their release behaviors, leading to the deviation from the Higuchi equation.

In Figure 7, the cumulative drug release of $\mathrm{NaBS}, \mathrm{NaSA}$, NaMAS, and NaLS after 7 days are $61.3 \%, 56.8 \%, 37.7 \%$, and $\sim 100 \%$ respectively. The changing trend of the $D$ values in Table 3 follows the same order as in pure water: NaLS > NaBS > NaSA > NaMAS. Therefore, the salicylates (NaSA and NaMAS) are relatively more suitable for controlled release by the HAEHFM.

\section{Conclusions}

The model drugs including salycilates (NaSA and NaMAS) and aromatic monoacids with similar structures $(\mathrm{NaBS}$ and NaLS) can be effectively loaded onto the hybrid anion 


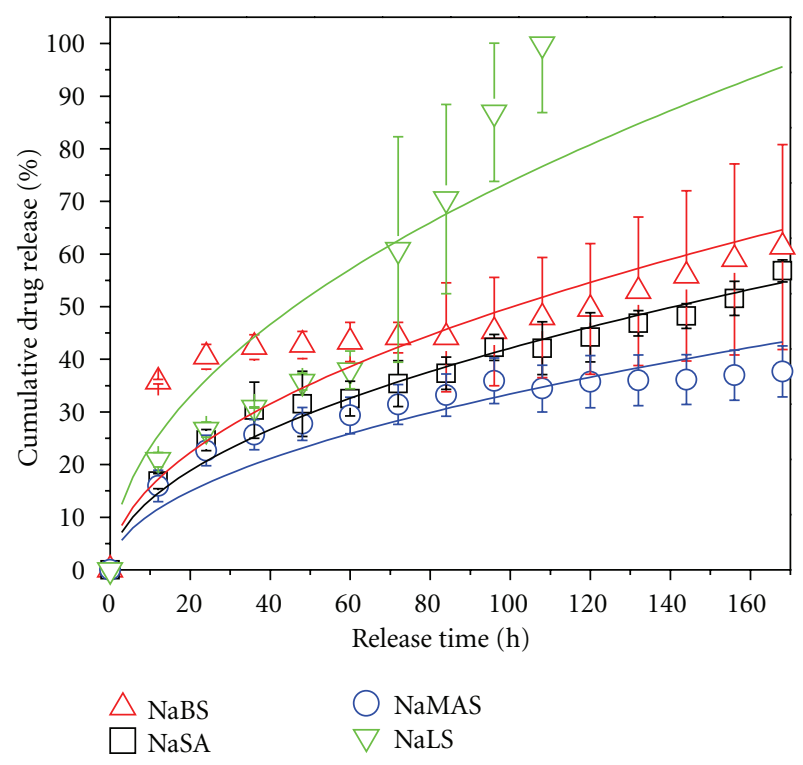

FIGURE 7: Release behaviors of different drugs from HAEHFM in a simulated body fluid at $37^{\circ} \mathrm{C}$.

exchange hollow fiber membrane (HAEHFM) with the $q_{m}$ value of $1.2-2.5 \mathrm{mmol} / \mathrm{g}$ dry membrane. The salycilates perform excellent release behavior (37.7\% NaMAS and 56.8\% NaSA released in simulated body fluid during 7 days). The physicochemical characteristics of drugs are crucial to the interaction patterns between the drugs and the membrane. Although the electrostatic interaction appears to play an important role between the anionic drugs and the anion exchange membrane, the nonelectrostatic interactions (hydrophobic interactions and hydrogen bonding) are also very significant to the drug release performances. Additionally, hydrogen bonding between the drugs and the membrane shows strong $\mathrm{pH}$ dependence. For example, the hydrogen bonding between NaMAS and the membrane can be disturbed in basic condition, resulting into an increase in the diffusion coefficient ( $D$ value) of NaMAS at $\mathrm{pH}$ 9.0. Thus, to optimize the release performance of the drug delivery system, the release environment, such as the salt concentrations and $\mathrm{pH}$ values, should be selected according to the physicochemical properties of the drug.

\section{Acknowledgments}

The authors gratefully acknowledge the financial support in part from the National Natural Science Foundation of China (Nos. 21025626 and 20974106), the National Basic Research Program of China (No. 2009CB623403), and the Natural Science Foundation of Anhui Province (No. 11040606M37).

\section{References}

[1] M. M. Bonvin and M. M. de Bertorello, "In vitro drug release from chitosan membranes-study of the mechanisms of permeation," Polymer Bulletin, vol. 32, no. 1, pp. 69-75, 1994.
[2] S. A. Burns, R. Hard, W. L. Hicks et al., "Determining the protein drug release characteristics and cell adhesion to a PLLA or PLGA biodegradable polymer membrane," Journal of Biomedical Materials Research Part A, vol. 94, no. 1, pp. 27-37, 2010.

[3] S. P. Schwendeman, G. L. Amidon, M. E. Meyerhoff, and R. J. Levy, "Modulated drug release using iontophoresis through heterogeneous cation-exchange membranes: membrane preparation and influence of resin cross-linkage," Macromolecules, vol. 25 , no. 9, pp. 2531-2540, 1992.

[4] A. Narebska, S. Koter, and W. Kujawski, "Irreversible thermodynamics of transport across charged membranes. Part I: macroscopic resistance coefficients for a system with nafion 120 membrane," Journal of Membrane Science, vol. 25, no. 2, pp. 153-170, 1985.

[5] Y. E. Fang, Q. Cheng, and X. B. Lu, "Kinetics of in vitro drug release from chitosan/gelatin hybrid membranes," Journal of Applied Polymer Science, vol. 68, no. 11, pp. 1751-1758, 1998.

[6] R. M. P. da Silva, S. G. Caridade, J. San Roman, J. F. Mano, and R. L. Reis, "Transport of small anionic and neutral solutes through chitosan membranes: dependence on cross-linking and chelation of divalent cations," Biomacromolecules, vol. 9, no. 8, pp. 2132-2138, 2008.

[7] H. N. Pei, X. G. Chen, Y. Li, and Y. Z. Hui, "Characterization and ornidazole release in vitro of a novel composite film prepared with chitosan/poly(vinyl alcohol)/alginate," Journal of Biomedical Materials Research Part A, vol. 85, no. 2, pp. 566-572, 2008.

[8] K. Kontturi, P. Pentti, and G. Sundholm, "Polypyrrole as a model membrane for drug delivery," Journal of Electroanalytical Chemistry, vol. 453, no. 1-2, pp. 231-238, 1998.

[9] S. Akerman, K. Akerman, J. Karppi et al., "Adsorption of drugs onto a poly(acrylic acid) grafted cation-exchange membrane," European Journal of Pharmaceutical Sciences, vol. 9, no. 2, pp. 137-143, 1999.

[10] S. Akerman, B. Svarfvar, K. Kontturi et al., "Influence of ionic strength on drug adsorption onto and release from a poly(acrylic acid) grafted poly(vinylidene fluoride) membrane," International Journal of Pharmaceutics, vol. 178, no. 1, pp. 67-75, 1999.

[11] L. Zhang, T. W. Xu, and Z. Lin, "Controlled release of ionic drug through the positively charged temperature-responsive membranes," Journal of Membrane Science, vol. 281, no. 1-2, pp. 491-499, 2006.

[12] K. Hanninen, A. M. Kaukonen, T. Kankkunen, and J. Hirvonen, "Rate and extent of ion-exchange process: the effect of phy-sico-chemical characteristics of salicylate anions," Journal of Controlled Release, vol. 91, no. 3, pp. 449-463, 2003.

[13] N. Wang, C. M. Wu, Y. Y. Cheng, and T. W. Xu, "Organicinorganic hybrid anion exchange hollow fiber membranes: a novel device for drug delivery," International Journal of Pharmaceutics, vol. 408, no. 1-2, pp. 39-49, 2011.

[14] T. Jaskari, M. Vuorio, K. Kontturi, A. Urtti, J. A. Manzanares, and J. Hirvonen, "Controlled transdermal iontophoresis by ion-exchange fiber," Journal of Controlled Release, vol. 67, no. 2-3, pp. 179-190, 2000.

[15] T. Jaskari, M. Vuorio, K. Kontturi, J. A. Manzanares, and J. Hirvonen, "Ion-exchange fibers and drugs: an equilibrium study," Journal of Controlled Release, vol. 70, no. 1-2, pp. 219-229, 2001.

[16] K. R. Hanninen, A. M. Kaukonen, L. S. Murtomaki, and J. T. Hirvonen, "Effect of ion-exchange fiber structure on the binding and release of model salicylates," Journal of Pharmaceutical Sciences, vol. 94, no. 8, pp. 1772-1781, 2005. 
[17] E. A. Braude and F. C. Nachod, Determination of Organic Structures by Physical Methods, Academic Press, New York, NY, USA, 1955.

[18] I. Raskin, "Role of salicylic acid in plants," Annual Review of Plant Physiology and Plant Molecular Biology, vol. 43, no. 1, pp. 439-463, 1992.

[19] W. Lund, The Pharmaceutical Codex, Principles and Practice of Pharmaceutics, The Pharmaceutical Press, London, UK, 25th edition, 1994.

[20] R. W. Sabnis, Handbook of Acid-Base Indicators, Taylor \& Francis Group, San Francisco, Calif, USA, 2008.

[21] N. Wang, C. M. Wu, Y. H. Wu, and T. W. Xu, "Hybrid anion exchange hollow fiber membranes through sol-gel process of different organic silanes within BPPO matrix," Journal of Membrane Science, vol. 363, no. 1-2, pp. 128-139, 2010.

[22] I. Langmuir, "The constitution and fundamental properties of solids and liquids. Part I. Solids," The Journal of the American Chemical Society, vol. 38, no. 2, pp. 2221-2295, 1916.

[23] W. I. Higuchi, "Analysis of data on the medicament release from ointments," Journal of Pharmaceutical Sciences, vol. 51, pp. 802-804, 1962.

[24] M. L. Glowka, D. Martynowski, and K. Kozlowska, "Stacking of six-membered aromatic rings in crystals," Journal of Molecular Structure, vol. 474, no. 1-3, pp. 81-89, 1999.

[25] V. S. Sumi, R. Kala, R. S. Praveen, and T. P. Rao, "Imprinted polymers as drug delivery vehicles for metal-based anti-inflammatory drug," International Journal of Pharmaceutics, vol. 349 , no. $1-2$, pp. 30-37, 2008.

[26] T. Kankkunen, I. Huupponen, K. Lahtinen et al., "Improved stability and release control of levodopa and metaraminol using ion-exchange fibers and transdermal iontophoresis," European Journal of Pharmaceutical Sciences, vol. 16, no. 4-5, pp. 273-280, 2002.

[27] O. L. Sprockel, M. Sen, P. Shivanand, and W. Prapaitrakul, "A melt-extrusion process for manufacturing matrix drug delivery systems," International Journal of Pharmaceutics, vol. 155, no. 2, pp. 191-199, 1997.

[28] D. Fitzpatrick and J. Corish, "Release characteristics of anionic drug compounds from liquid crystalline gels: I: passive release across non-rate-limiting membranes," International Journal of Pharmaceutics, vol. 301, no. 1-2, pp. 226-236, 2005.

[29] K. Hanninen, A. M. Kaukonen, T. Kankkunen, and J. Hirvonen, "Rate and extent of ion-exchange process: the effect of physico-chemical characteristics of salicylate anions," Journal of Controlled Release, vol. 91, no. 3, pp. 449-463, 2003.

[30] J. L. Wood, "pH-controlled hydrogen-bonding," Biochemical Journal, vol. 143, no. 3, pp. 775-777, 1974. 

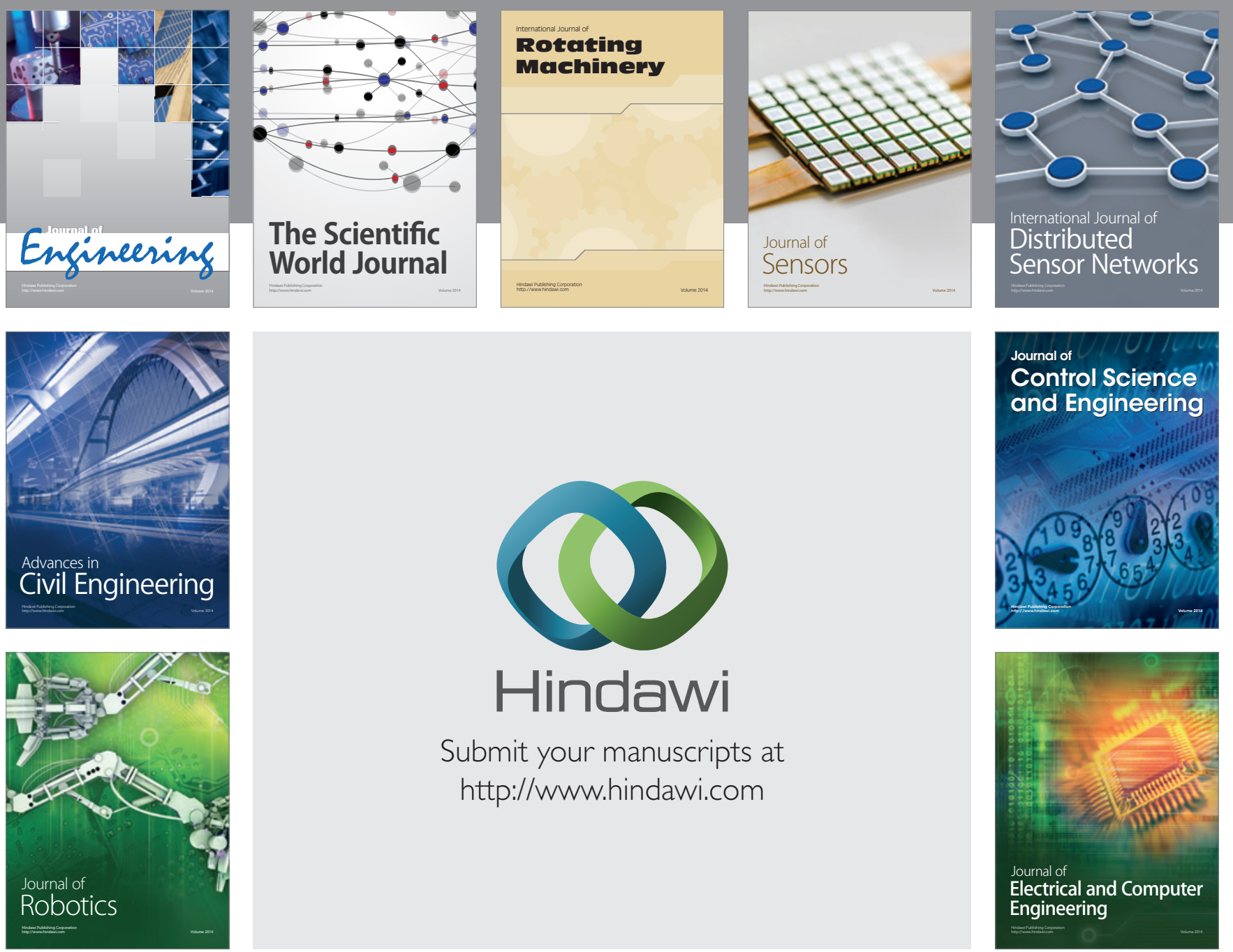

Submit your manuscripts at

http://www.hindawi.com
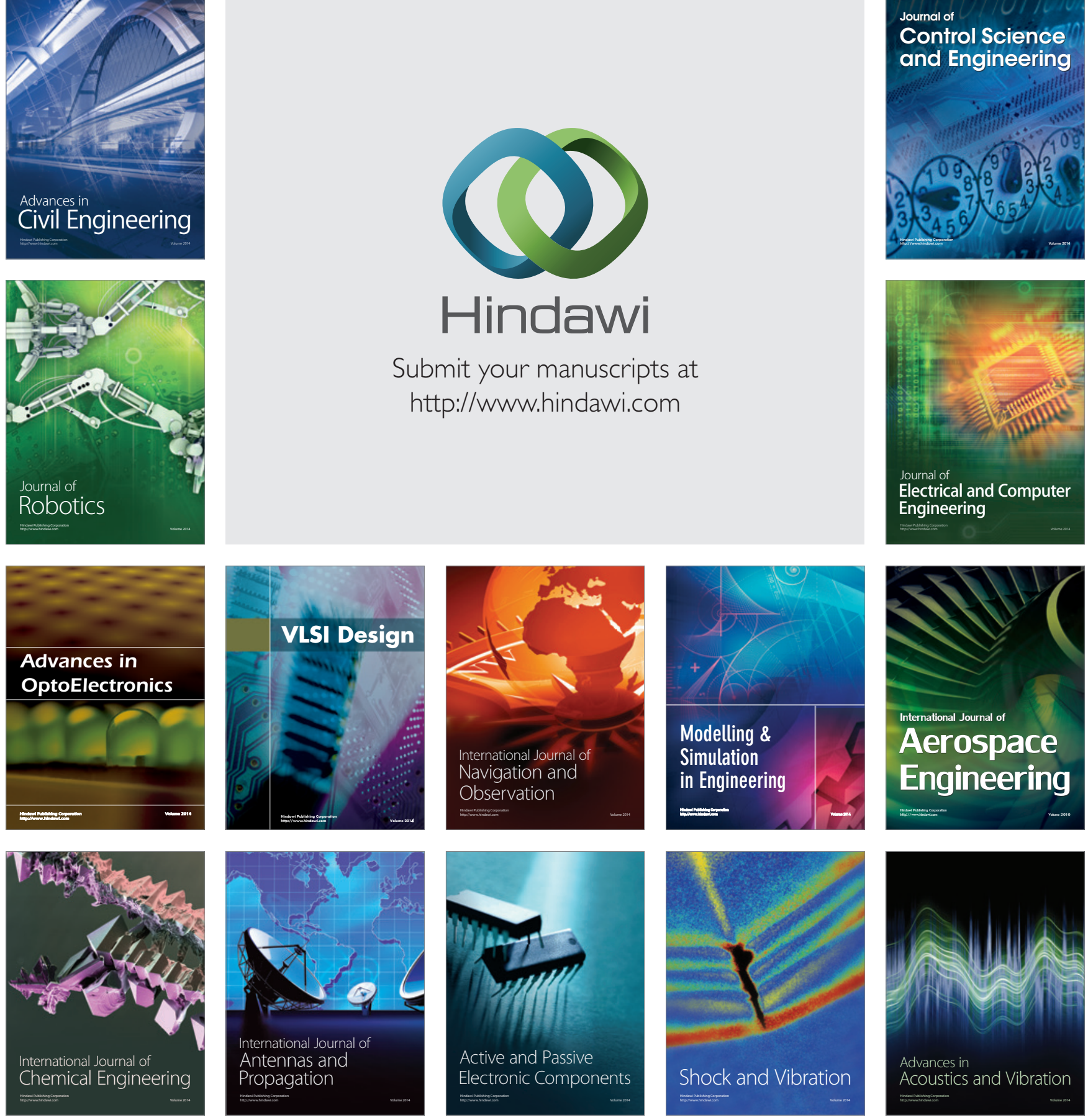